\title{
An Efficient Radio Frequency Channel Distribution in 5G Heterogeneous Cellular Networks for Avoiding Cross-Tier Interference in Macro and Small Cells
}

\author{
Ahmed Qaddus* (Bahria University, Islamabad, Pakistan), \\ Shahzad Hassan (Bahria University, Islamabad, Pakistan), \\ Abid Ali Minhas (Al Yamamah University, Riyadh, Kingdom of Saudi Arabia)
}

\begin{abstract}
Recently, the need for user data rate traffic has increased for running high-bandwidth applications. Therefore, the way forward lies in 5G heterogeneous cellular networks. The 5G network is comprised of two network hierarchies. As the first hierarchy, there are MBSs with large macro cells for macro users. As the second hierarchy, there are FBSs referred to as small cells for femto users. The 5G networks encourage the use of large macro and small cells for efficient utilisation and distribution of channel resources. In this study, the authors have proposed an efficient RF channel distribution mechanism on the basis of the current SINR levels of FUEs and MUEs. On the basis of the users' present SINR levels, the channels will be allocated by the central MBS to MUEs and FUEs via FBSs. The major obstacle in RF channel allocation to FUEs and MUEs is cross-tier interference at the downlink channel at the MUEs and FUEs from the transmitted signals of MBSs and FBSs. In this study, an efficient RF channel allocation scheme is proposed on the basis of channel modelling constraints, which will minimise the cross-tier interference at the downlink channel at the MUEs and FUEs during RF channel allocation to FUEs and MUEs present in the same coverage area.
\end{abstract}

Keywords - 5G heterogeneous cellular networks; Centralised MBS; Femto base station (FBS); Femto user equipment (FUE); Macro base station (MBS); Macro cell; Radio frequency (RF); Macro user equipment (MUE); Received signal strength indication (RSSI); Signal-to-interference-plus-noise ratio (SINR); Small cell.

\section{INTRODUCTION}

With the remarkable increase in the user traffic demands for running high-bandwidth applications, where every user applies for a dedicated bandwidth depending on the data rate required for playing real-time applications. The available cellular technologies like $2 \mathrm{G}, 3 \mathrm{G}$ and $4 \mathrm{G}$ do not fully meet the increasing user bandwidth demands. Therefore, in order to cope with the current circumstances, $5 \mathrm{G}$ heterogeneous cellular networks are proposed for addressing the current deficiencies like network capacity [1]-[3].

In order to offer high-bandwidth application services to customers, the network has to optimise its RF channel resources efficiently. By efficient distribution of RF channel resources to users based on their application usage, different bandwidth can be allocated for the voice, data and video users. By adopting this mechanism, the cellular network technology can optimise its RF resources inventively, in a proficient way [1]-[3].
Further, by the dynamic distribution of RF channel resources, the network operators can easily utilise the unused channel bandwidth portion as the users are allocated channels on the basis of their required current application bandwidth needs. Therefore, by using the dynamic distribution of RF channel resources, different bandwidths can be allocated according to the requirements of the various voice, data and live streaming video applications [1]-[3].

\section{5G HETEROGENEOUS CELLULAR NETWORKS}

For meeting the user broad-bandwidth demands, 5G heterogeneous cellular networks are proposed, which will be the future of networks supporting a high data broadband mobile cellular networks. The $5 \mathrm{G}$ technology is expected to be operational by the year 2020 with a high data rate transfer speed. In addition to these high data rate services, $5 \mathrm{G}$ will also be able to provide scalability, connectivity and energy-efficient mobile cellular networks [3]-[9].

The predecessors of $5 \mathrm{G}$ cellular technologies like $2 \mathrm{G}, 3 \mathrm{G}$ and $4 \mathrm{G}$ cannot cope with increasing user data rate demands, as they can offer limited data rates for user applications as shown in Table I. Therefore, in the previous cellular technologies there was no room for improvement left to increase the bandwidth due to their operation in a limited licensed spectrum. These cellular networks were not designed to operate in the unlicensed ISM $2.4 \mathrm{GHz}$ and $5 \mathrm{GHz}$ bands [3]-[9].

TABLE I

Data Rates of Predecessor Cellular TeChNOLOgies [3]

\begin{tabular}{|l|l|}
\hline Cellular technology & Supported data rate \\
\hline $2 \mathrm{G}$ & $64 \mathrm{kbps}$ \\
\hline $3 \mathrm{G}$ & $2 \mathrm{Mbps}$ \\
\hline $4 \mathrm{G}$ & $50 \mathrm{Mbps}$ to $100 \mathrm{Mbps}$ \\
\hline
\end{tabular}

$5 \mathrm{G}$ heterogeneous cellular networks, for better utilisation of the RF resources, recommend the use of small cells and macro cells. The small cells are served by femto base stations and the large macro cells are served by macro base stations. For the

* Corresponding author.

E-mail: ahmedqaddus@gmail.com 
distribution of RF channel resources, 5G heterogeneous cellular networks opt for a centralised approach. Here, a centralised macro base station of the macro cell is responsible for the RF channel resource distribution to macro users served by macro base stations and femto users served by femto base stations. Further, the centralised macro base station of macro cell distribute the RF channel resources to femto users of small cells located currently in the vicinity of the macro cell base station's coverage region as shown in Figure 1 [10]-[13].

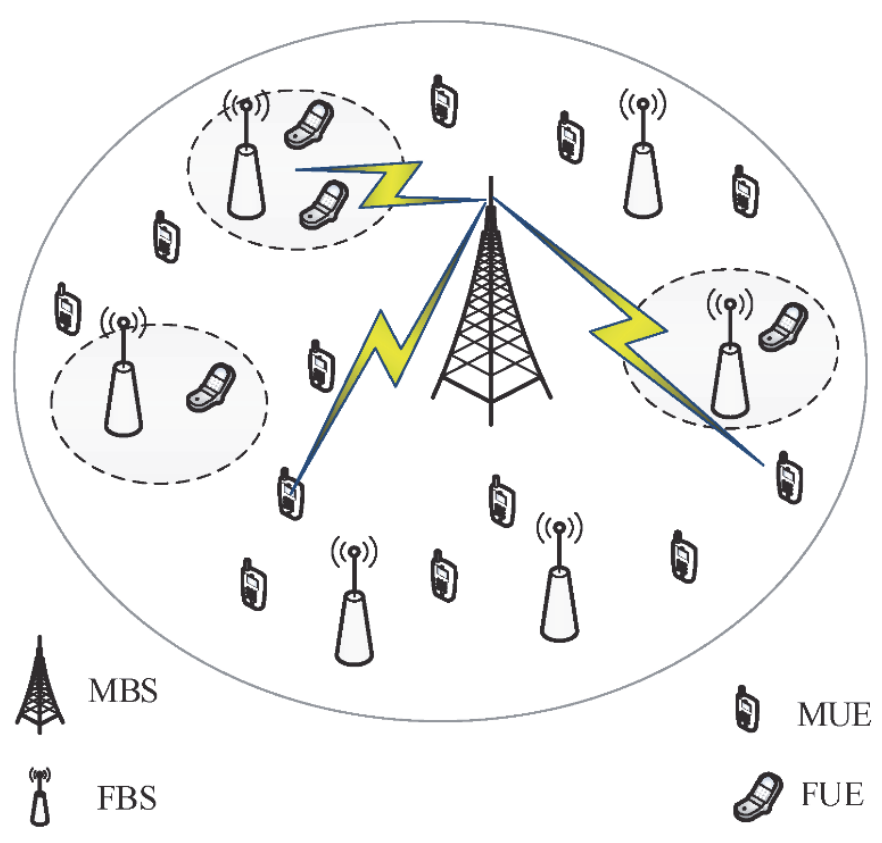

Fig. 1. Macro cell and small cells in a 5G heterogeneous network.

$5 \mathrm{G}$ networks are designed to operate in both unlicensed spectrum ISM bands like $2.4 \mathrm{GHz} / 5 \mathrm{GHz}$ bands and the licensed spectrum of $24-86 \mathrm{GHz}$ bands. The frequencies operating in the $24-86 \mathrm{GHz}$ bands are also referred to as the millimetre waves ( $\mathrm{mmW}$ ) technology. The RF spectrum of the millimetre waves $(\mathrm{mmW})$ lies between $30 \mathrm{GHz}$ and $300 \mathrm{GHz}$. The millimetre waves ( $\mathrm{mmW}$ ) offer a higher bandwidth, which enables users to run high-data-rate applications in Gbps. Multiple-input multiple-output (MIMO) antenna diversities will be used in $5 \mathrm{G}$ heterogeneous cellular networks to avoid the phenomenon of absorption losses encountered at this higher level of the frequencies spectrum. Figure 2 shows a deployment scenario of future $5 \mathrm{G}$ heterogeneous networks where the network is becoming still denser due to the large amount of user traffic and the bandwidth demands. Here, millimetre wave $(\mathrm{mmW})$ frequencies with small cells are deployed, for better frequency reuse and enhancing network capacity [14]-[21].

In addition to the multiple-input multiple-output (MIMO) antenna diversities, $5 \mathrm{G}$ heterogeneous cellular networks will offer a faster data transfer and guaranteed channel availability to small cell users and Macro cell users. 5G intends to use beam-forming techniques where RF coverage of antenna is extensive only in a certain area of cell coverage where the user is currently located and traffic unburdening on to unlicensed ISM 2.4 GHz/ 5GHz frequency band spectrum [14]-[21].

\section{Towards 5G Networks}
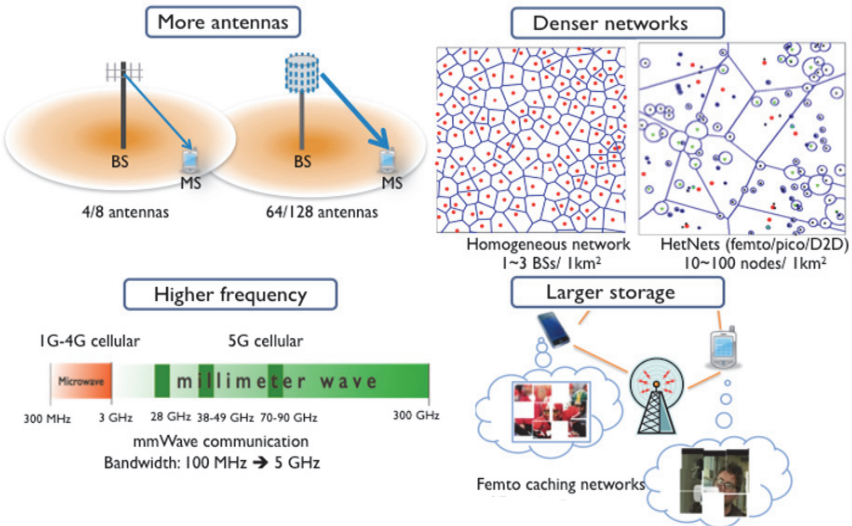

Fig. 2. Deployment of future 5G heterogeneous cellular network.

The predecessors of $5 \mathrm{G}$ heterogeneous cellular network technologies like the $2 \mathrm{G}, 3 \mathrm{G}$ and $4 \mathrm{G}$ cellular networks could not operate in the unlicensed ISM frequency band spectrum and were not capable of operating in the full-duplex communication mode of operation. In contrast, 5G heterogeneous cellular networks are designed to operate in the full-duplex communication mode of operation. By using this full-duplex communication mode, the channels can be used concurrently for the function of transmission and reception to/from the MUE present in the macro cell and the FUE present in the small cell. The recommended network operating parameters of $5 \mathrm{G}$ heterogeneous cellular networks are shown in Table II [3], [14]-[21].

TABLE II

Suggested 5G Hetnets Working Parameters [31]

\begin{tabular}{|l|l|}
\hline Parameter & Suggested Performance \\
\hline Network Capacity & $\begin{array}{l}10000 \text { times the capacity of } \\
\text { a current network }\end{array}$ \\
\hline Peak data rate & $10 \mathrm{Gbps}$ \\
\hline Cell edge rate & $100 \mathrm{Mbps}$ \\
\hline Latency & $<1 \mathrm{~ms}$ \\
\hline
\end{tabular}

According to the proposed 5G standard, the network capacity will be enhanced; it will be more than 10000 times what the user is currently receiving from $4 \mathrm{G}$ networks. The potential cause of the increase in the network capacity is related to the low latency levels - less than $1 \mathrm{~ms}$. Due to the low latency levels, the obtained SINR levels increase, which causes a further decrease in the bit error rates. The quality of the received signal and the RSSI values are enhanced due to the decrease in the bit error rates, caused by extensive beamforming phenomenon of the multiple-input multiple-output (MIMO) antenna diversities. In 5G heterogeneous cellular networks, small cells known as femto cells will be deployed near the premises of the femto users inside the coverage area of 
the larger macro cells. The purpose of the deployment of small cells near the premises of the femto users is to accommodate high-data-rate real-time traffic applications. Table III shows the recommended cell radius and transmit power of the macroand small-cell base stations in 5G HetNets [3], [14]-[21].

TABLE III

Cell Radil AND Transmit Power LeVels in 5G HetNets [3]

\begin{tabular}{|c|c|c|}
\hline \multicolumn{3}{|c|}{ Cell radius \& transmit power } \\
\hline Cell type & Cell radius & $\begin{array}{c}\text { Transmit } \\
\text { power }\end{array}$ \\
\hline Macro & $1 \mathrm{~km}$ to $16 \mathrm{~km}$ & $40 \mathrm{~W} \max$ \\
\hline Small & $10 \mathrm{~m}$ to $50 \mathrm{~m}$ & $200 \mathrm{~mW} \max$ \\
\hline
\end{tabular}

\section{PROBLEM STATEMENT}

Cross-tier interference is considered to be one of the critical issues for HetNets where MUEs and FUEs experience unwanted interference on the downlink channel from the transmitting signals of MBSs and FBSs belonging to a different tier, which causes severe performance degradation. Since $5 \mathrm{G}$ cellular architecture will follow HetNets, the issue of cross-tier interference will need to be addressed in an efficient way to obtain real benefits from 5G networks [19]-[23], [25], [26].

\section{SUGGESTED ELUCIDATION}

The authors of this study have acclaimed the use of developing an efficient and robust channel scheduling optimisation scheme to reduce cross-tier interference at MUEs and FUEs in downlink channel from their surrounding MBSs and FBSs in HetNets. Further, we will try to meet minimum QoS criteria for macro and femto users belonging to their respective serving macro and femto cells. Secondly, we will form an optimisation problem and use one of the evolutionary algorithms (e.g. the genetic algorithm, particle swarm optimisation and ant colony optimisation) for an optimal solution.

\section{SYSTEM MODEL}

We consider a two-tier macro and femto deployment in the $5 \mathrm{G}$ heterogeneous network as depicted in Figure 3, where FBSs with a cell radius of $\mathrm{Rf}$ are randomly deployed inside the centralised MBS station with a cell radius of $\mathrm{Rm}$. The channel assignment in MUEs and FUEs inside the femto cell in $5 \mathrm{G}$ heterogeneous networks is formulated as $k=\{1,2,3, \ldots, N\}$, $F(\mathrm{FUE})=\{1,2,3, \ldots, F\}, \dot{M}(\mathrm{MUE})=\{1,2,3, \ldots, M\}$, $\dot{B}($ femto cell $)=\{1,2,3, \ldots, B\}$. For efficient use of spectrum and to address spectral scarcity, the FUEs and MUEs share the same poll of the spectrum when performing communication over the same channel. However, such optimal sharing leads to cross-tier interference issues. In this study, we will be primarily focusing on cross-tier interference, which is the primary cause of interference in the FUEs and MUEs deployed in HetNets, which are getting their services from both the MBSs and the FBSs at the same time while using the same frequency channel. The sharing of the same frequency channel by FUEs and MUEs make the cross-tier interference more probable, which is something that needs to be addressed more thoroughly than the co-tier interference, which can be decreased by an efficient reuse mechanism and orthogonal resource allocation to femto cells.

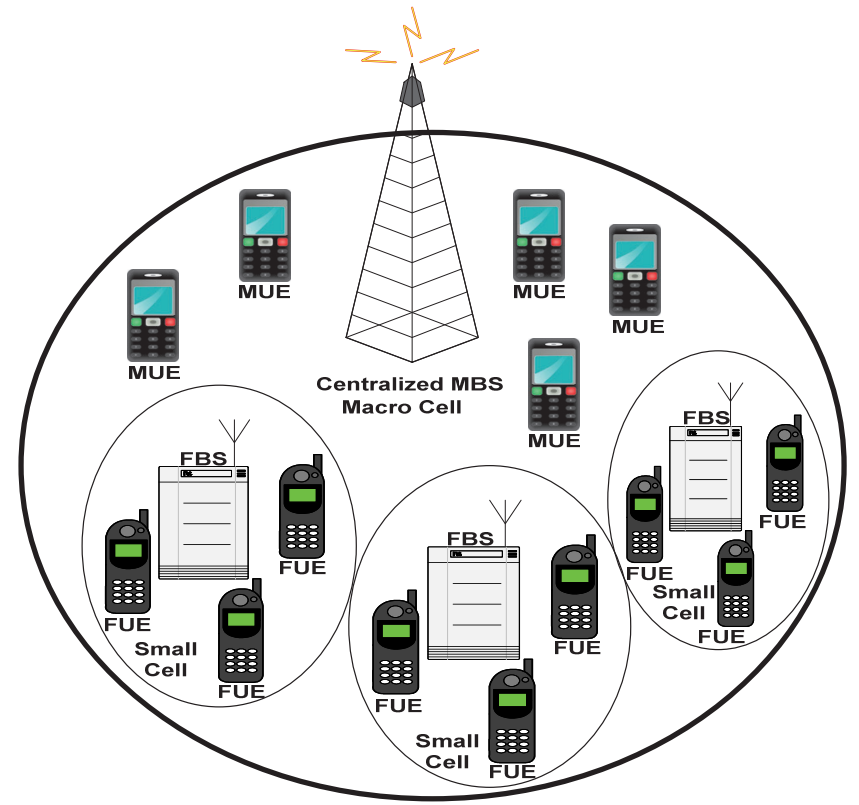

Fig. 3. System Model for $5 \mathrm{G}$ heterogeneous cellular network.

To address cross-tier interference, all the MUEs and FUEs are allocated channel resources through a central entity, which not only provides interference-minimised channel allocation but also ensures minimal QoS. The QoS service parameter depends on the macro and femto user equipment SINR levels, which should be greater than the SINR threshold of the femto and macro base station to ensure a good Quality of Service (QoS) for running high-bandwidth applications without delay or jitter. Further, the SINR levels at the macro and femto user equipment can be enhanced by using beam-forming antenna techniques where RF coverage of antenna is extensive only in a certain area of cell coverage where the user is currently located. We have assumed that all the macro and femto base stations and units of user equipment are equipped with beamforming antennas as proposed by the 5G standard [14]-[21], which radiate $\mathrm{RF}$ power within the angle of $360^{\circ}$ equally in all the directions of the cell coverage area. In our system model, we have assumed that the FBSs and MBSs present in the $5 \mathrm{G}$ networks are connected to a centralised MBS via the Internet, fibre optics and microwave backhaul links.

Further, we have considered that perfect synchronisation is present between the MBSs and FBSs inside the coverage region of $5 \mathrm{G}$ heterogeneous cellular networks. The phenomenon of interference will not occur during the scheduling cycles and the RF power of the MUEs and FUEs will be kept constant. Crosstier interference occurs at the downlink channel where MUE is interfered by the transmitted RF signals $\left(\mathrm{G}^{\circ}\right)$ of FBS and FUE is interfered by the transmitted RF signals $\left(\mathrm{H}^{\circ}\right)$ of MBS, with the relevant abbreviations depicted in Table I \&IV. Due to the phenomenon of cross-tier interference the user applications, the data rate will be affected drastically due to the low levels of SINR, which will result in High BERs, which in turn will lead to delay and jitter in video application streaming. 
TABLE IV

USED In THE CHANNEL Allocation Algorithm

\begin{tabular}{|c|c|}
\hline Symbols & Meanings \\
\hline$C^{j}$ & Capacity of MUE \\
\hline$C^{i}$ & Capacity of FUE \\
\hline$F$ & Total number of FUE \\
\hline$M$ & Total number of MUE \\
\hline$U^{f}$ & FUE present in the area \\
\hline$U^{m}$ & MUE present in the area \\
\hline$P^{m}$ & Transmit power of MBS \\
\hline$P^{f}$ & Transmit power of FBS \\
\hline$H$ & Downlink channel of MUE \\
\hline$G$ & Downlink channel of FUE \\
\hline$H^{\circ}$ & Interference from MBS to FUE \\
\hline$G^{\circ}$ & Interference from FBS to MUE \\
\hline$X^{m}$ & Transmitted signal of MBS \\
\hline$X^{f}$ & Transmitted signal of FBS \\
\hline$Z^{m}, Z^{f}$ & Additive white Gaussian noise (AWGN) \\
\hline$\sigma^{n^{\wedge} 2}$ & Noise variance \\
\hline$\gamma(j)$ & SINR at MUE \\
\hline$\gamma(i)$ & SINR at FUE \\
\hline$\alpha$ & SINR threshold of FBS \\
\hline$\beta$ & SINR threshold of MBS \\
\hline$Y^{m}, Y^{f}$ & Interference at MUE and FUE \\
\hline$b$ & Femto cell \\
\hline$k$ & Channel subscript \\
\hline$i$ & FUE subscript \\
\hline$j$ & MUE subscript \\
\hline
\end{tabular}

\section{CHANNEL MODEL}

The channel model used in our study is depicted in Figure 4 with the interfered signals from an MBS and an FBS to an MUE and an FUE. We designate the downlink channel for the MBSto-MUE link by $\mathrm{H}$ and the one for the FBS-to-FUE link by $\mathrm{G}$. Here, 5G heterogeneous cellular networks encounter two key types of interference: the interference from the MBS to the FUE is designated by $\mathrm{H}^{\circ}$ and the interference from the FBS to the MUE is designated by $\mathrm{G}^{\circ}$. During the downlink reception of $\mathrm{RF}$ channel $\mathrm{H}$ from the MBS to the MUE, there is a probable cause of interference present at the MUE from the interfered signals $\mathrm{G}^{\circ}$ from the neighbouring FBS located in the close vicinity of FUE.

The second interference occurs at downlink reception of RF channel $\mathrm{G}$ from the FBS to the FUE; here, interfered signals $\mathrm{H}^{\circ}$ from the central MBS is received at the FUE present inside the coverage region umbrella of the central MBS. FUE We use indices $\mathrm{i}$ and $\mathrm{j}$ for the FUE and MUE respectively, which are being served at a given transmit time interval (TTI) and with a given physical resource. We are using the terms 'PRB' and 'channel' interchangeably.

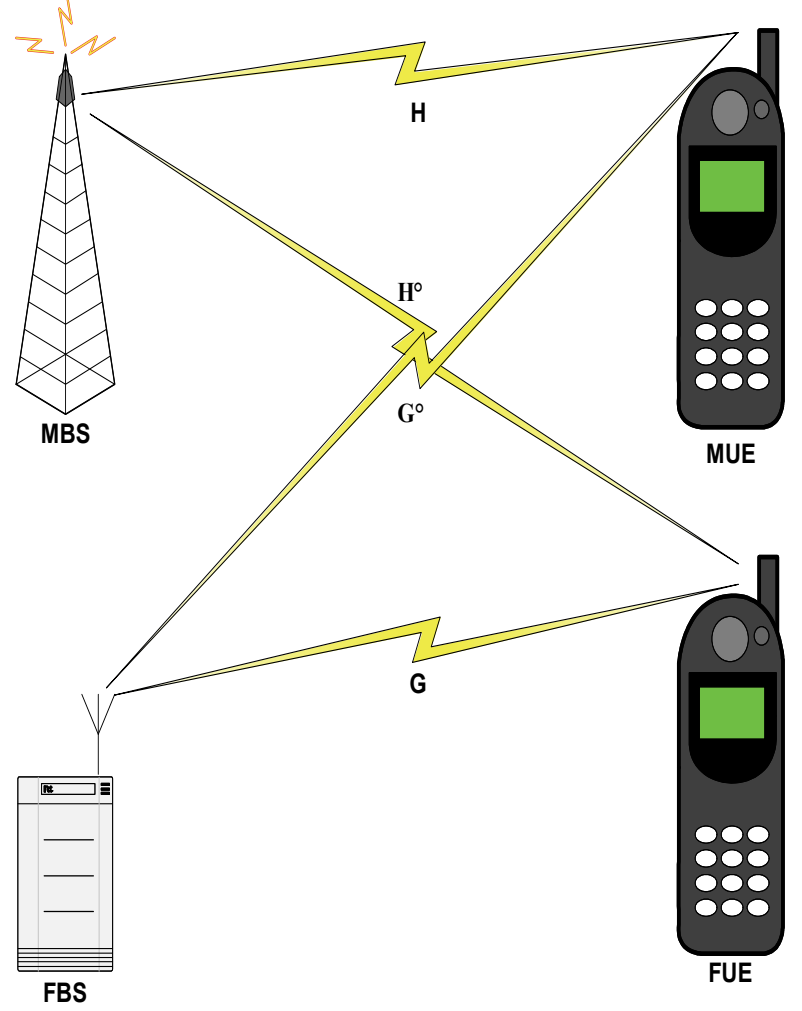

Fig. 4. Downlink TX channel model in a $5 \mathrm{G}$ network.

The process of RF channel resource allocation to MUEs and FUEs from the centralised MBSs and FBSs is illustrated in a block diagram shown in Figure 5. The RF channel resources are allocated to the MUEs and FUEs based on their current SINR levels from the centralised MBSs and FBSs.

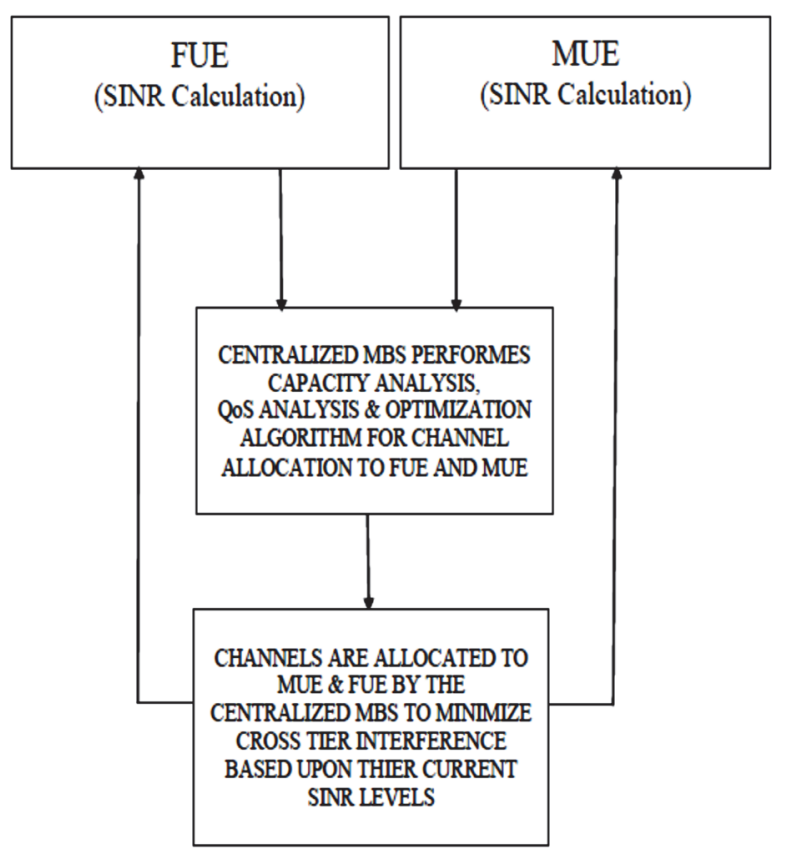

Fig. 5. Block diagram of channel allocation. 
The signal-to-interference-plus-noise ratio (SINR) [28] in macro and femto user equipment is calculated as follows:

$$
\begin{gathered}
\gamma\left(j^{k}\right)=\frac{P^{m^{\wedge} 2}\left\|H^{k}\right\|^{2}}{\sigma^{n^{\wedge 2}}+P^{f^{\wedge} 2} \Sigma_{k}\left\|G^{\circ k}\right\|^{2}} ; \\
\gamma\left(i^{k}\right)=\frac{P^{f^{\wedge 2}}\left\|G^{k}\right\|^{2}}{\sigma^{n^{\wedge}}+P^{m^{\wedge} 2} \sum_{k}\left\|H^{\circ k}\right\|^{2}} ;
\end{gathered}
$$

Here $\sigma^{n \wedge 2}$ is the noise variance and the main focus is on optimizing spectral efficiency, each BS schedules the user with the highest SINR [28]. The receive channel capacity of FUE and MUE can be calculated according to Eqs. (3) and (4), respectively.

$$
\begin{gathered}
C_{k}^{b, i}=\mathrm{B}_{\log _{2}\left[1+\gamma\left(i^{k}\right)\right]} \\
C_{k}^{j}=\mathrm{B} \log _{2}\left[1+\gamma\left(j^{k}\right)\right] .
\end{gathered}
$$

Based on the above discussion, the channel allocation in the heterogeneous networks is formulated as the following optimisation problem:

$$
\operatorname{Max} \sum_{b=1}^{B} \sum_{i=1}^{F} \sum_{K=1}^{N} C_{k}^{b, i} x_{k}^{b, i}+\sum_{j=1}^{M} \sum_{K=1}^{N} C_{k}^{j} x_{k}^{j}(5)
$$

The SINR of the macro and femto User Equipment should be equal to or better than the SINR threshold of the femto base station and the macro base station for good quality of service (QoS) of applications of a high bandwidth without delay or jitter. Further maximisation of throughput and the SINR results in the reduction or elimination of inter-cell interference in macro and femto cells as shown in equation (6) and (7).

(Calculated from Eqs. (1) and (2).)

$$
\begin{aligned}
& \gamma\left(i^{k}\right) \geq \alpha \\
& \gamma\left(j^{k}\right) \geq \beta \\
& x_{k}^{b, i}=\left\{\begin{array}{c}
1 ; \text { if } \gamma\left(i^{k}\right) \geq \alpha ; \\
0 ; \text { otherwise; }
\end{array}\right. \\
& x_{k}^{j}=\left\{\begin{array}{l}
1 ; \text { if } \gamma\left(j^{k}\right) \geq \beta ; \\
0 ; \text { otherwise; }
\end{array}\right. \\
& x_{k}^{b, i} \neq x_{k}^{j}, \forall i \neq j \\
& \sum_{b=1}^{B} \sum_{i=1}^{F} \sum_{K=1}^{N} x_{K}^{b, i} \leq 1 ; \\
& \sum_{\mathrm{j}=1}^{M} \sum_{K=1}^{N} x_{\mathrm{k}}^{j} \leq 1 ;
\end{aligned}
$$

$x_{k}^{b, i}=1$, if $i$-th has a better SINR, the $\mathrm{k}$-th channel is allocated to femto user equipment $i$-th and $x_{k}^{b, i}=0$, if it remains out due to a poor SINR as indicated in Eq. (8).

$x_{k}^{j}=1$, if $j$-th has a better SINR, the k-th channel is allocated to macro user equipment $j$-th and $x_{k}^{j}=0$, if it remains out due to poor SINR as indicated in Eq. (9).

The objective function in (5) is to maximise the sum throughput of femto and macro users in the heterogeneous network. The SINR requirements of the macro user equipment (MUE) and the femto user equipment (FUE) are mentioned in $(6,7)$ whereas $\alpha$ and $\beta$ are the SINR thresholds for the macro and femto networks. The RF channel allocation strategy for allocation of RF channels to macro user equipment (MUE) and femto user equipment (FUE) is based on three constraints. Constraint 1 from Eqs. (5) to (9) shows the goal to maximise the capacity or the data rate of the heterogeneous networks on the basis of the macro and femto user equipment SINR without degrading the quality of service $(\mathrm{QoS})$ of the macro and femto user equipment.

Constraint 2 from equation (10) shows the goal to provide not more than one channel to a single unit of macro or femto user equipment. Equation (10) shows an assumption that a single macro or femto user will be allocated only a single channel physical resource block (PRB). By maximizing the throughput that is achieved in (5), we assume that the inter-cell interference is reduced significantly or is not present. Therefore, the assumption in (10) can be carried forward and only a single channel will be allocated to macro and femto user equipment.

Constraint 3 from equations (11) to (12) shows the goal to provide one channel per one unit of macro and femto user equipment. Here, we assumed that only one channel $k$ from the channel pool can be assigned to the $i$-th femto user and the $j$-th macro user.

\section{PATH LOSS (PL) MODELS}

The path loss (PL) models [27] used in our research work are based on the cross-tier macro and femto base station coverage area for indoor and outdoor deployments. Here in the first case the FUEs and MUEs present in indoor buildings are getting coverage from the FBSs deployed indoors and the MBSs deployed outdoors. In the second case, the FUEs and MUEs present in the outdoor environment are getting coverage from the FBS deployed indoors and the MBS deployed outdoors.

\section{A. MBS (outdoor) to MUE (outdoor):}

$$
15.3+37.6 \lg (d)
$$

\section{B. FBS (indoor) to FUE (outdoor):}

$$
\begin{gathered}
38.46+20 \lg (d)+0.7 d_{\text {indoor }}+L_{\mathrm{w}} \\
\left(d_{\text {indoor }}=0.5 \mathrm{~dB}, \text { wall penetration } L_{\mathrm{w}}=10 \mathrm{~dB}\right) .
\end{gathered}
$$

C. MBS (outdoor) to MUE (indoor):

$$
15.3+37.6 \lg (d)+L_{\mathrm{w}}\left(L_{\mathrm{w}}=10 \mathrm{~dB}\right) .
$$




\section{FBS (indoor) to FUE (indoor):}

$$
38.46+20 \lg (d)+0.7 d_{\text {indoor }}\left(d_{\text {indoor }}=0.5 \mathrm{~dB}\right) .
$$

Here $\mathrm{d}$ is the distance between the MBS and the FBS and between the MUE and the FUE, WP represents the loss due to wall penetration, which is assumed to be $10 \mathrm{~dB}$. The shadowing factor of $8 \mathrm{~dB}$ and $4 \mathrm{~dB}$ is also considered for indoor and outdoor conditions.

$$
\begin{aligned}
& H=10^{-P L / 10} \\
& G=10^{-P L / 10}
\end{aligned}
$$

Further in our research we have used channel gain $\mathrm{G}$ and $\mathrm{H}$ mentioned in Eqs. (17) and (18), which is greatly affected by the path loss conditions, depending on the different outdoor and indoor deployments.

\section{RF CHANNEL RESOURCE ALLOCATION}

In our study we have selected evolutionary algorithms like particle swarm optimisation for allocation of RF channels resource to FUEs and MUEs present in the MBS and FBS coverage area of $5 \mathrm{G}$ heterogeneous cellular networks. The RF channels will be allocated to the FUEs and MUEs present in the $5 \mathrm{G}$ heterogeneous cellular networks on the basis of their present signal-to-interference-plus-noise ratio (SINR) levels [28]. SINR is inversely proportional to the bit error rates (BER) and latency levels and directly proportional to capacity. In order to run high-capacity bandwidth data rate applications, SINR levels at the receiver side should be higher for better performance [29], [30].

\section{Simulation OUTCOMES}

In this study, the authors have validated different MUE and FUE scenarios with simulations. Here MUEs and FUEs are scattered at different locations from the MBSs and FBSs as shown in Figs. 6 and 7. As the MUE and FUE move away from the MBS and FBS coverage region, the RSSI values decrease, which results in lesser values of SINR and higher latency levels at the FUEs and MUEs.

The authors have simulated SINR and capacity levels at the FUEs and MUEs which are interfered by the neighbouring FBSs and MBSs present inside the 5G heterogeneous cellular networks architecture. Figures 8 and 9 show that higher SINR levels at the receiver side result in a higher capacity for supporting high-data-rate bandwidth applications. The channel capacity of the individual MUE and FUE depends on the SINR levels. The greater the received SINR levels of the MUE and FUE, the greater would the channel capacity of the individual MUE and FUE be. Figs. 10 and 11 show the channel capacity of individual MUEs and FUEs based upon their present SINR levels.

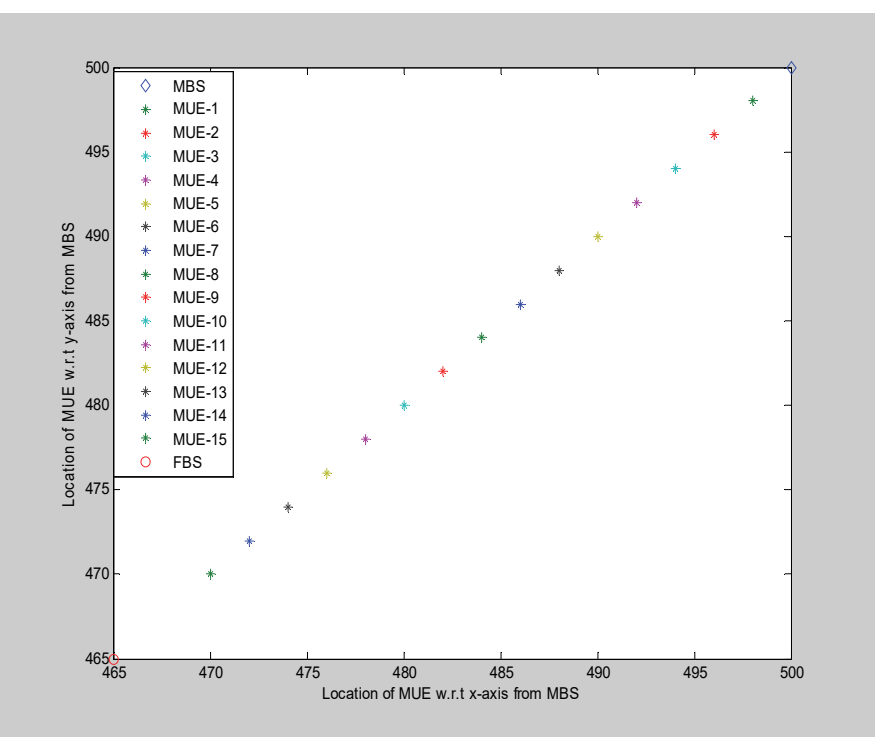

Fig. 6. Location of MUEs w.r.t. MBSs.

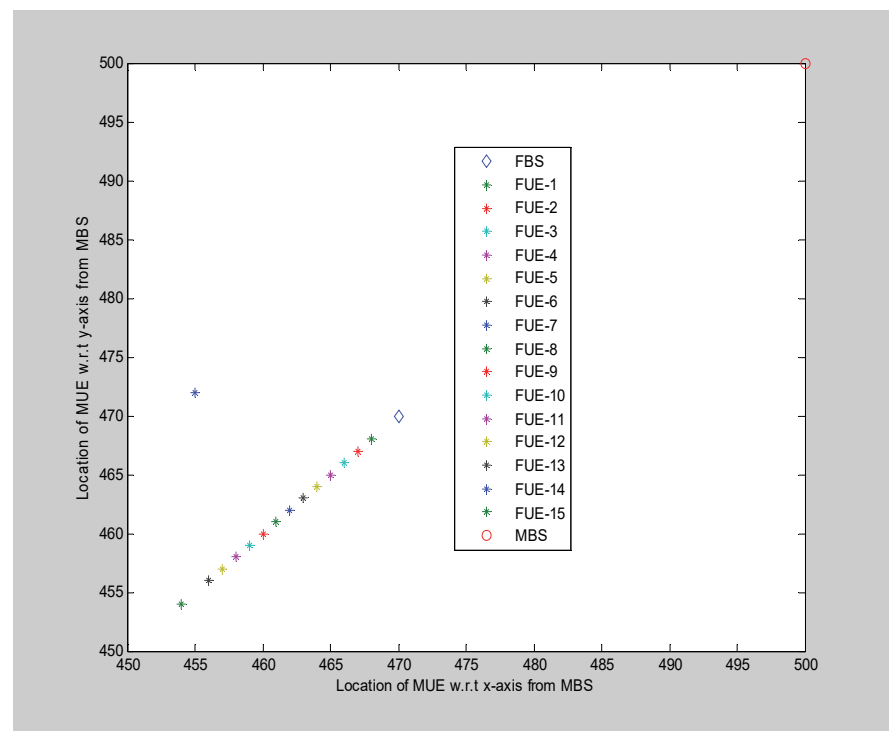

Fig. 7. Location of FUEs w.r.t. FBSs.

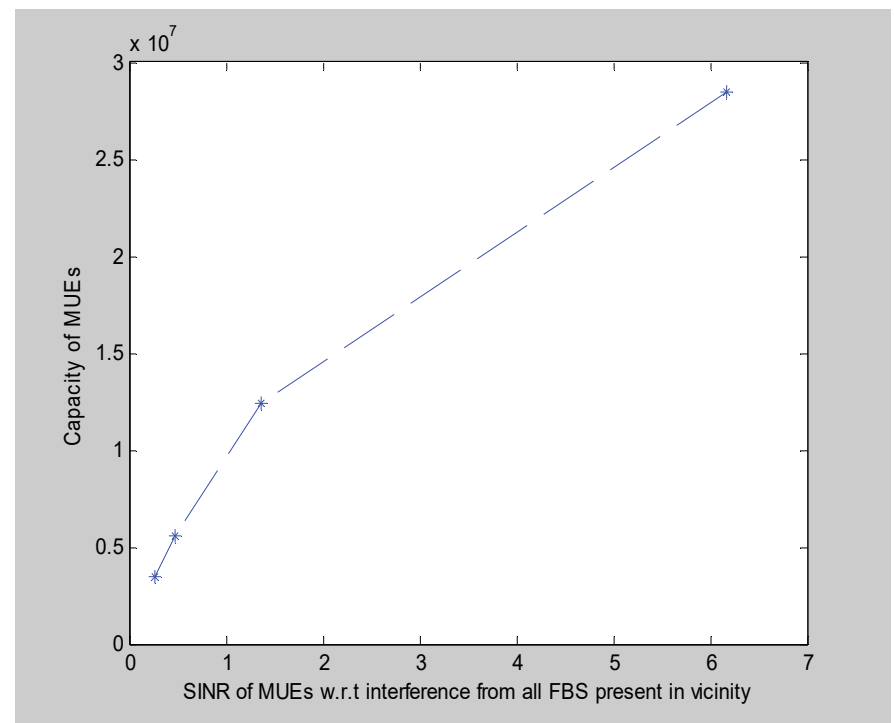

Fig. 8. SINR \& Capacity of MUEs w.r.t. interference from FBS. 
2018, vol. 14 , no. 1

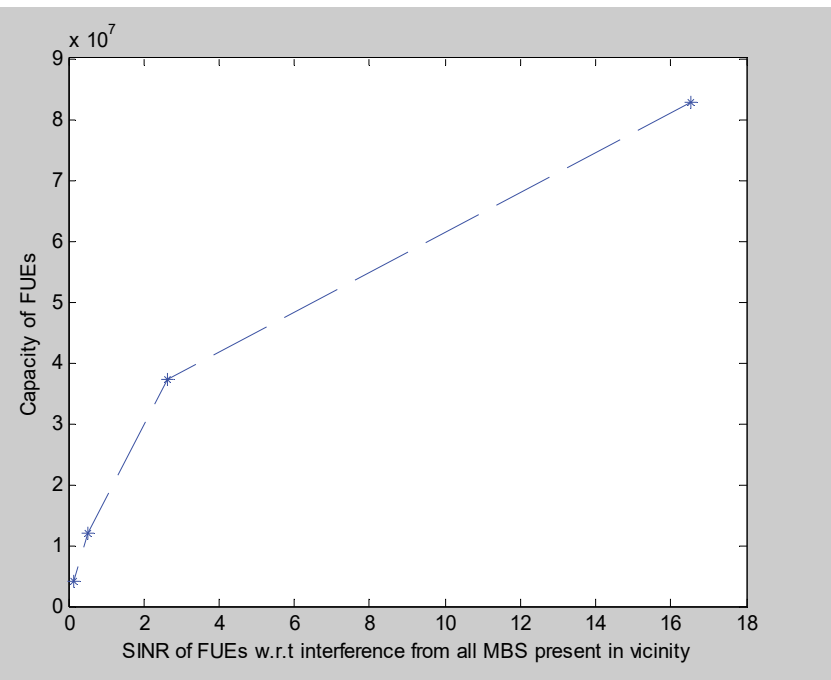

Fig. 9. SINR \& Capacity of FUEs w.r.t. interference from MBSs.

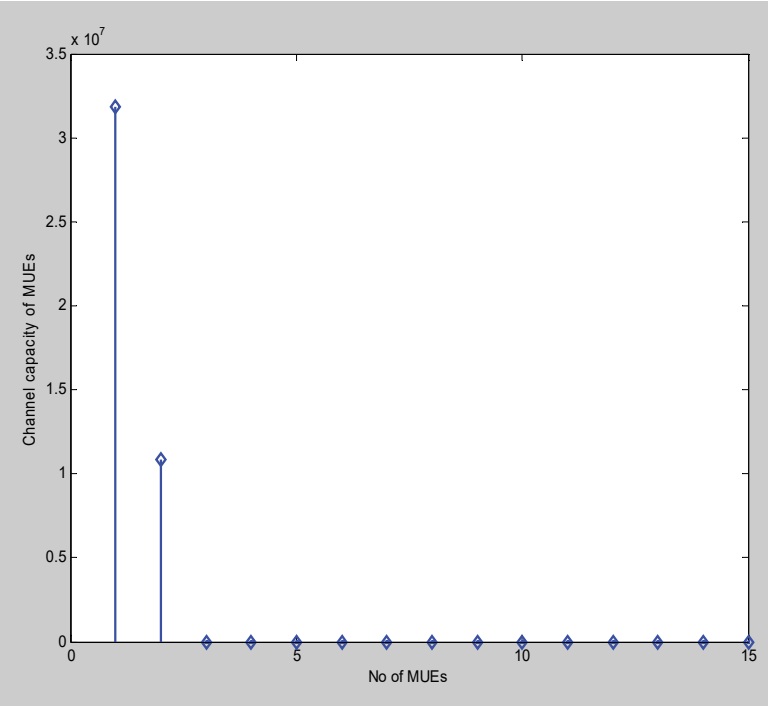

Fig. 10. Channel capacity of MUEs.

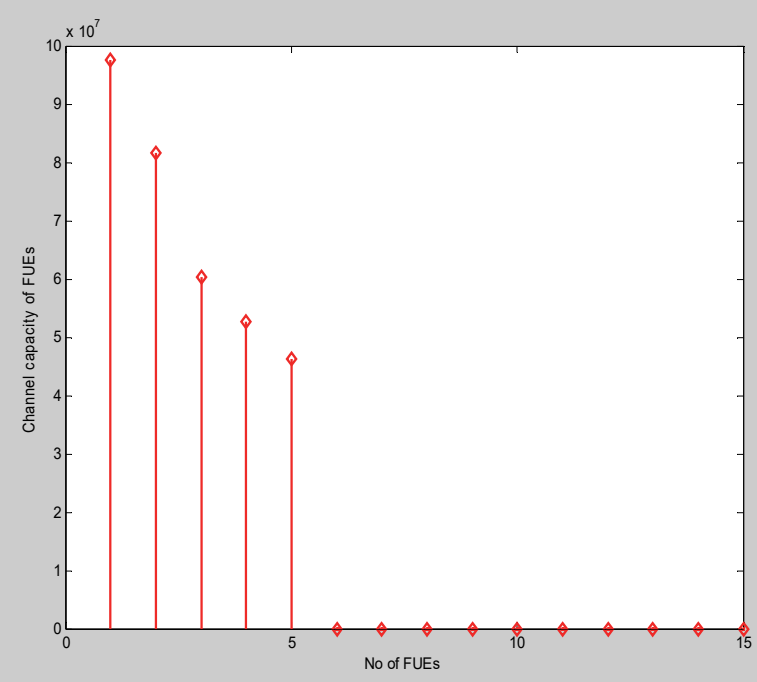

Fig. 11. Channel capacity of FUEs.
Further for channel optimisation, the authors in this study have used the particle swarm optimisation (PSO) algorithm. Figs. 12 to 14 show the results for the PSO algorithm from 50 to 1700 iterations, which shows the maximum number of channel capacity (the objective function in equation 5) on the $y$-axis; the $x$-axis shows the number of iterations.

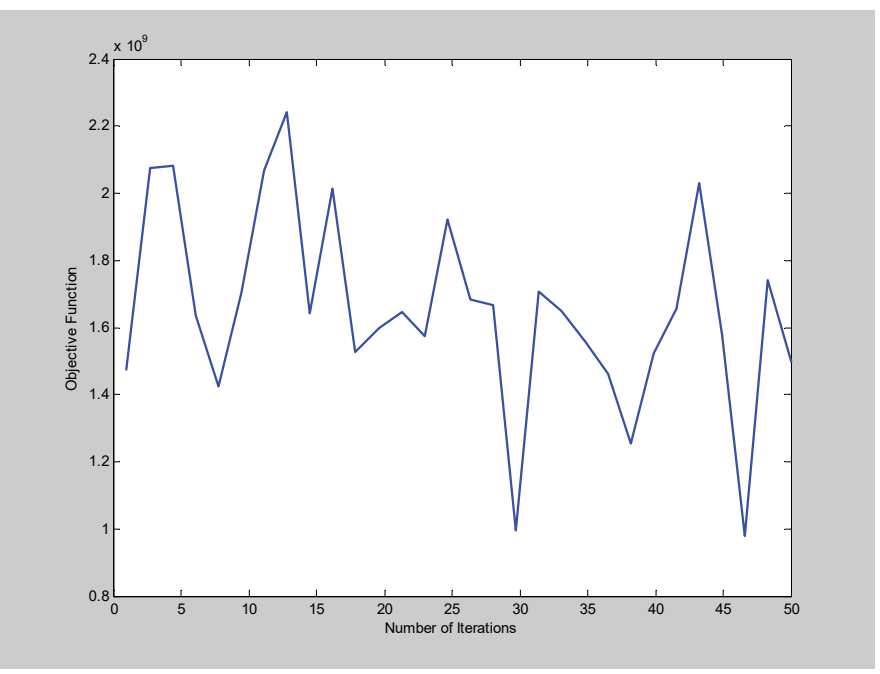

Fig. 12. Channel capacity of FUEs.

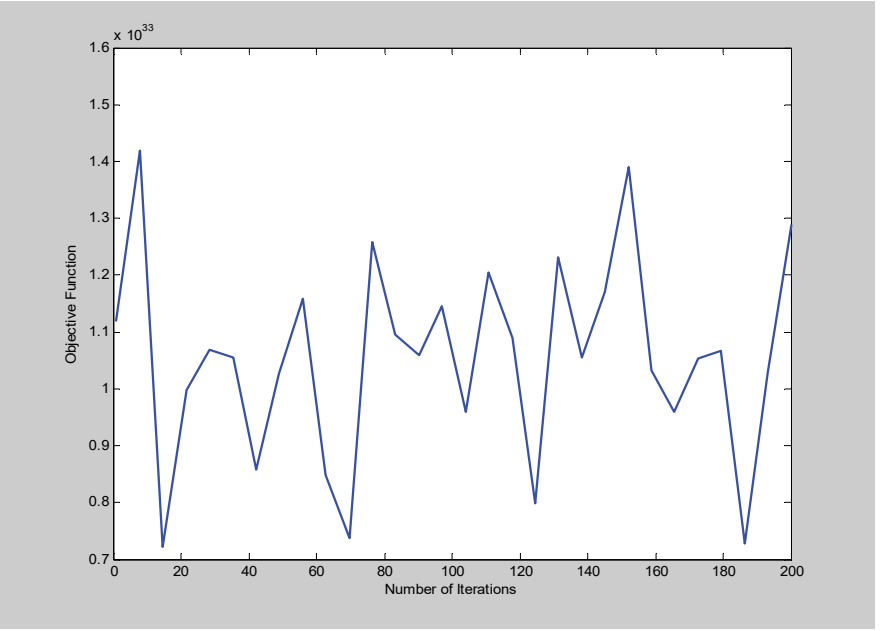

Fig. 13. Channel capacity of FUEs.

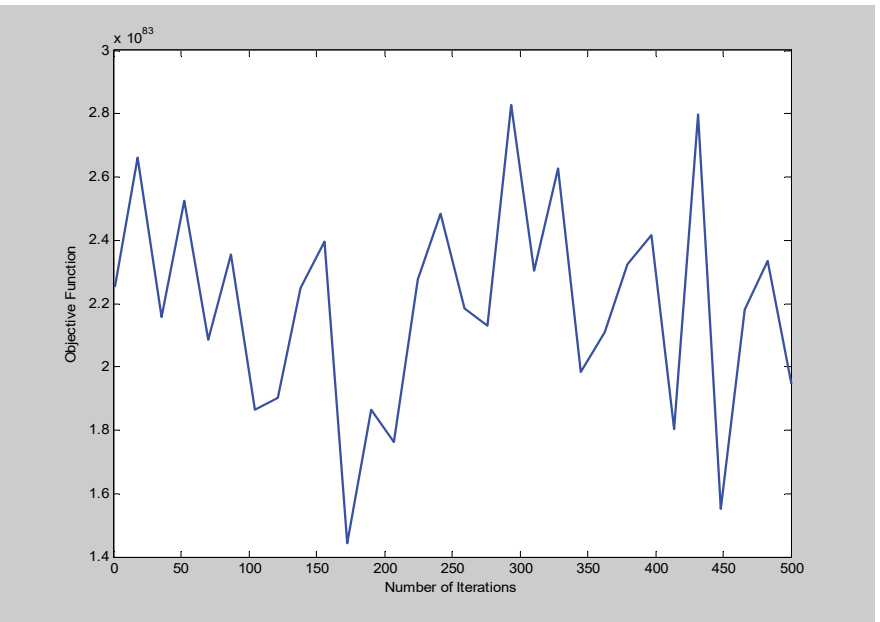

Fig. 14. Channel capacity of FUEs. 


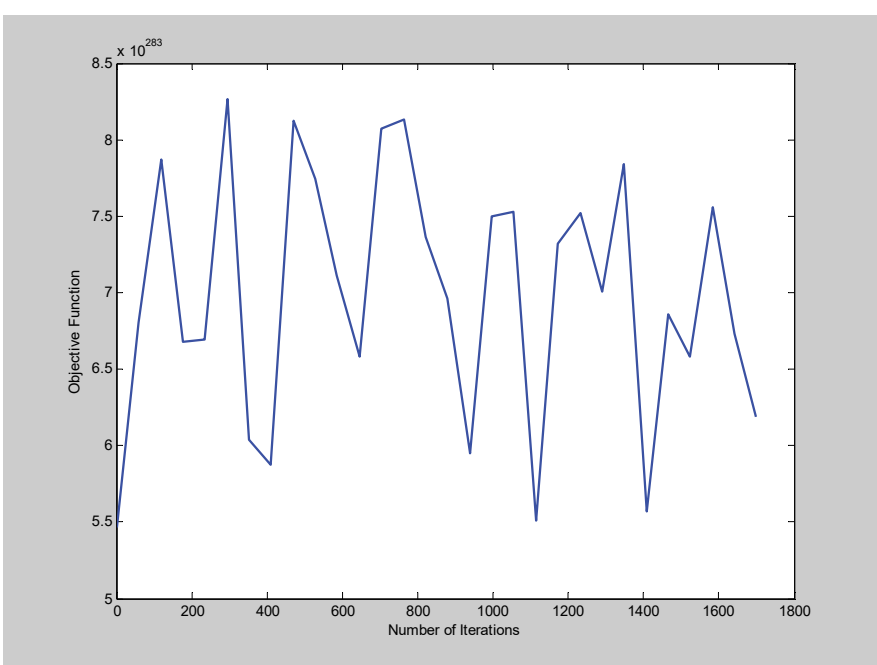

Fig. 15. Channel capacity of FUEs.

\section{CONCLUSION}

Here the authors have suggested elucidation of developing an efficient and robust channel scheduling optimisation scheme, which will try to minimise the cross-tier interference in $5 \mathrm{G}$ cellular heterogeneous networks. In this study, we have addressed the use of efficient channel allocation scheduling schemes, by adopting three key channel modelling constraints, which have not been addressed previously. By efficient channel scheduling, we have minimised the cross-tier interference issues at MUEs and FUEs on the downlink channel from the transmitting signals of MBSs and FBSs, which further results in low bit error rates (BER) and high signal-to-interferenceplus-noise ratio SINR values. Further, the authors have also suggested in this study that higher SINR levels at the FUEs and MUEs will result in a higher capacity for supporting high-datarate bandwidth applications. Therefore we can adopt 5G heterogeneous cellular networks to craft a future high-speed cellular data network, which will be cost-effective and efficient with regard to RF channel resource distribution.

\section{REFERENCES}

[1] J. Gozalvez, "5G Worldwide Developments," IEEE Vehicular Technology Magazine, vol. 12, issue 1, pp. 4-11, Mar. 2017. https://doi.org/10.1109/mvt.2016.2641138

[2] S. Zhang, Q. Wu, S. Xu, and G. Y. Li "Fundamental Green Tradeoffs: Progresses, Challenges and Impacts of 5G Networks," IEEE Communication Surveys \& Tutorials, vol. 19, no. 1, pp. 33-56, 2017. https://doi.org/10.1109/comst.2016.2594120

[3] R. N. Mitra and D. P. Agrawal, "5G Technology: A Survey", Information and Communication Technology Express, vol. 1, no. 3, pp. 132-137, Dec. 2015. https://doi.org/10.1016/j.icte.2016.01.003

[4] M. Mueck, E. C. Strinati, I.-G. Kim, A. Clemente, J.-B. Dore, A. De Domenico, T. Kim, T. Choi, H. K. Chung, G. Destino, A. Parssinen, A. Pouttu, M. Latva-aho, N. Chuberre, M. Gineste, B. Vautherin, M. Monnerat, V. Frascolla, M. Fresia, W. Keusgen, T. Haustein, A. Korvala, M. Pettissalo, and O. Liinamaa, "5G Champion - Rolling out 5G in 2018," in 2016 IEEE Globecom Workshops (GC Wkshps), Dec. 2016. https://doi.org/10.1109/glocomw.2016.7848798

[5] Q. C. Li, H. Niu, A. T. Papathanassiou, and G. Wu, "5G Network Capacity Key Elements and Technologies," IEEE Vehicular Technology Magazine, vol. 9, no. 1, pp. 71-78, Mar. 2014. https://doi.org/10.1109/mvt.2013.2295070

[6] I. Tzelatis and K. Berberidis, "Cross-Tier Interference Management Schemes in Cognitive Heterogeneous Networks," EURASIP Journal on Wireless Communications and Networking, vol. 2014, no. 1, Sep. 2014. https://doi.org/10.1186/1687-1499-2014-143
[7] Q. Zhao and D. Grace, "Transfer Learning for QoS Aware Topology Management in Energy Efficient 5G Cognitive Radio Networks," in Proc. 1st International Conference on $5 G$ for Ubiquitous Connectivity (5GU), 2014. https://doi.org/10.4108/icst.5gu.2014.258141

[8] S. Aleksic, "Towards Fifth-Generation (5G) Optical Transport Networks," in 2015 17th International Conference on Transparent Optical Networks (ICTON), Jul. 2015. https://doi.org/10.1109/icton.2015.7193532

[9] V. Yazıcı, C. Kozat, and M. Sunay, "A New Control Plane for 5G Network Architecture With a Case Study on Unified Handoff, Mobility, and Routing Management," IEEE Communications Magazine, vol. 52, no. 11, pp. 76-85, Nov. 2014. https://doi.org/10.1109/mcom.2014.6957146

[10] A. Damnjanovic, J. Montojo, Y. Wei, T. Ji, T. Luo, M. Vajapeyam, T. Yoo, O. Song, and D. Malladi, "A Survey on 3GPP Heterogeneous Networks," IEEE Wireless Communications, vol. 18, no. 3, pp. 10-21, Jun. 2011. https://doi.org/10.1109/mwc.2011.5876496

[11] M. I. Sanchez, A. Asadi, M. Draxler, R. Gupta, V. Mancuso, A. Morelli, A. de la Oliva, and V. Sciancalepore, "Tackling the Increased Density of 5G Networks; The CROWD Approach," in 2015 IEEE 81 ${ }^{\text {st }}$ Vehicular Technology Conference, May 2015. https://doi.org/10.1109/vtcspring.2015.7146122

[12] H. Tabassum, M. Z. Shakir, and M.-S. Alouini, "Area Green Efficiency (AGE) of Two Tier Heterogeneous Cellular Networks," in 2012 IEEE Globecom Workshop on Heterogeneous and Small Cell Networks (HetSNets), Dec. 2012. https://doi.org/10.1109/glocomw.2012.6477629

[13] A. Qaddus, A. Ishfaq, and A. Mustafa, "A Novel Solution Based on Cognitive Heterogeneous Networks for Removing Black Spots in TETRA Coverage Area," in 2013 5th International Conference on Information and Communication Technologies, Karachi, Dec. 14-15, 2013. https://doi.org/10.1109/icict.2013.6732777

[14] M. Newinger, A. Dotzler, and W. Utschick, "Interference Shaping for Device to Device Communication in Cellular Networks", in IEEE International Conference on Communications, London, Jun. 8-12. 2015. https://doi.org/10.1109/icc.2015.7248969

[15] E. Z. Tragos, S. Zeadally, A. G. Fragkiadakis, and V. A. Siris, "Spectrum Assignment in Cognitive Radio Networks: A Comprehensive Survey," IEEE Communications Surveys \& Tutorials, vol. 15, no. 3, pp. 11081135, 2013. https://doi.org/10.1109/surv.2012.121112.00047

[16] W. Nam, D. Bai, J. Lee, and I. Kang, "Advanced Interference Management for 5G Cellular Networks", IEEE Communications Magazine, vol. 52, no. 5, pp. 52-60, May 2014. https://doi.org/10.1109/mcom.2014.6815893

[17] C.-X. Wang, F. Haider, X. Gao, X.-H. You, Y. Yang, D. Yuan, H. Aggoune, H. Haas, S. Fletcher, and E. Hepsaydir, "Cellular Architecture and Key Technologies for 5G Wireless Communication Networks", IEEE Communications Magazine, vol. 52, no. 2, pp. 122-130, Feb. 2014. https://doi.org/10.1109/mcom.2014.6736752

[18] Q. C. Li, H. Niu, A. T. Papathanassiou, and G. Wu, "Edge Cloud and Underlay Networks: Empowering 5G Cell-Less Wireless Architecture," in European Wireless, Barcelona, Spain, May 14-16, 2014.

[19] M. Ahmed and J. Kim, "Context Aware Network-Assisted Hand-off Management and Interference Mitigation Scheme for Heterogeneous Networks", in 2014 IEEE Wireless Communications and Networking Conference (WCNC), Apr. 2014. https://doi.org/10.1109/wenc.2014.6952930

[20] D. Aziz, M. Mazhar, and A. Weber, "Multi User Inter Cell Interference Alignment in Heterogeneous Cellular Networks," in 2014 IEEE 79th Vehicular Technology Conference (VTC Spring), May 2014.

[21] J. Dommel, P.-P. Knust, L. Thiele, and T. Haustein, "Massive MIMO for Interference Management in Heterogeneous Networks," in IEEE 8th Sensor Array and Multichannel Signal Processing Workshop (SAM), Jun. 2014. https://doi.org/10.1109/sam.2014.6882398

[22] K. Bahmani, R. Bakhshi, E. A. Ince, and C. M. Yetis, "Interference Management in Two-Tier Heterogeneous Networks Using Blind Interference Alignment," in 2015 23nd Signal Processing and Communications Applications Conference (SIU), May 2015. https://doi.org/10.1109/siu.2015.7130188

[23] T. M. Ho, N. H. Tran, C. T. Do, S. M. A. Kazmi, E.-N. Huh, and C. S. Hong, "Power Control for Interference Management and QoS Guarantee in Heterogeneous Networks," IEEE Communications Letters, vol. 19, no. 8, Aug. 2015. https://doi.org/10.1109/lcomm.2015.2444844 
[24] A. Qaddus, A. A. Raza, and A. Mustafa, "Deploying Uninterrupted Wireless Communication Networks by Using Software Define Cognitive Radios (SDCR) and Time Division Duplex (TDD) Transmission Techniques in 5G Networks,” 2015 International Conference on Information and Communication Technologies (ICICT), Dec. 2015. https://doi.org/10.1109/icict.2015.7469586

[25] C.-H. Huang and C.-Y. Liao, "An Interference Management Scheme for Heterogeneous Network With Cell Range Extension," in 2011 13th AsiaPacific Network Operations and Management Symposium, Sep. 2011. https://doi.org/10.1109/apnoms.2011.6077021

[26] L. Yi and T. Cui, "Interference Mitigation Between Femtocell and Macro Cell," in Proc. 2011 IEEE International Conference on Electronics and Optoelectronics (ICEOE), Jul. 29-31, 2011. https://doi.org/10.1109/iceoe.2011.6013186

[27] C. Bouras, V. Kokkinos, K. Kontodimas, and A. Papazois, "A Simulation Framework for LTE-A Systems With Femtocell Overlays," in Proc. 7th ACM workshop on Performance monitoring and measurement of heterogeneous wireless and wired networks (PM2HW2N '12), Paphos, Cyprus, Oct. 21-22, 2012, pp. 85-90.

https://doi.org/10.1145/2387191.2387204

[28] H. Zhang, C. Jiang, X. Mao, and H.-H. Chen, "Interference-Limited Resource Optimization in Cognitive Femtocells With Fairness and Imperfect Spectrum Sensing," IEEE Transactions on Vehicular Technology, vol. 65, no. 3, pp. 1761-1771, Mar. 2016. https://doi.org/10.1109/tvt.2015.2405538

[29] R. Yankevych, "Newly Developed Mechanism of Signal-to-Noise Ratio Estimation", in 2015 16th International Conference on Computational Problems of Electrical Engineering (CPEE), Sep. 2015. https://doi.org/10.1109/cpee.2015.7333384

[30] M. Dryjanski and M. Szydelko, "A Unified Traffic Steering Framework for LTE Radio Access Network Coordination," IEEE Communication Magazine, vol. 54, no. 7, pp. 84-92, Jul. 2016. https://doi.org/10.1109/mcom.2016.7509383

[31] "5G Mobile Wireless Technology." [Online]. Electronics Notes. Available: http://www.radio-electronics.com/info/cellulartelecomms/5gmobile-cellular/technology-basics.php [Accessed: 25 June 2018].
Ahmed Qaddus received his B. sc. degree in telecommunications from SZABIST in 2008. Later he did his M. sc. in telecommunications \& networking from Bahria University Islamabad with a research paper in 2011 and won the Gold Medal. Currently, he is a Ph. D. student at Bahria University Islamabad. His research interests include communication and networks, wireless technologies, heterogeneous networks and spectrum management for macro and femtocell networks.

E-mail: ahmedqaddus@gmail.com

Dr. Shahzad Hassan is a Senior Assistant Professor at Department of Computer Engineering, Bahria University, Pakistan. He obtained his Ph.D.in Electronics and Information Engineering degree from Huazhong University of Science and Technology, Wuhan, Hubei, China and MS. Telecommunication Engineering from The University of Melbourne, Melbourne, Australia. E-mail: shassan.buic@bahria.edu.pk ORCID iD: https://orcid.org/0000-0002-4670-0438

Dr. Abid Ali Minhas is the Chairman of the Department of Computer Engineering at the College of Computer and Information Systems (CCIS) of Al Yamamah University. He did his Ph. D. in wireless sensor networks at Graz University of Technology, Graz, Austria, at the Department of Electrical Engineering. He won a Ph. D. scholarship from the Higher Education Commission (HEC) of Pakistan and completed his Ph. D. with the highest honours. He has been heading a research group at the Bahria University Wireless Research Centre (BUWRC). This group was engaged in activities related to wireless mobile communications/networks. He is the editor of the "Bahria University Journal of Information \& Communication Technology". E-mail:a_minhas@yu.edu.sa ORCID iD: https://orcid.org/0000-0002-7669-4008 